\title{
Irreducible coverings by cliques and Sperner's theorem
}

\author{
Ioan Tomescu \\ Faculty of Mathematics and Computer Science, \\ University of Bucharest, \\ Str. Academiei, 14 R-70109 Bucharest, Romania. \\ ioan@math.math.unibuc.ro
}

Submitted: September 29, 2002; Accepted: October 22, 2002.

MR Subject Classifications: 05C69, 05C35, 06A07

\begin{abstract}
In this note it is proved that if a graph $G$ of order $n$ has an irreducible covering of its vertex set by $n-k$ cliques, then its clique number $\omega(G) \leq k+1$ if $k=2$ or 3 and $\omega(G) \leq\left(\begin{array}{c}k \\ \lfloor k / 2\rfloor\end{array}\right)$ if $k \geq 4$. These bounds are sharp if $n \geq k+1$ (for $k=2$ or 3 ) and $n \geq k+\left(\begin{array}{c}k \\ k k / 2\rfloor\end{array}\right)$ (for $\left.k \geq 4\right)$.
\end{abstract}

Key Words: clique, irreducible covering, antichain, Sperner's theorem

\section{Definitions and preliminary results}

For a graph $G$ having vertex set $V(G)$ and edge set $E(G)$ a clique is a subset of vertices inducing a complete subgraph of $G$ which is maximal relative to set inclusion. The clique number of $G$, denoted $\omega(G)$, is the size of a largest clique in $G$ [1]. A $k$-clique is a clique containing $k$ vertices. A family of different cliques $c_{1}, c_{2}, \ldots, c_{s}$ of $G$ is a covering of $G$ by cliques if $\bigcup_{i=1}^{s} c_{i}=V(G)$. A covering $C$ of $G$ consisting of $s$ cliques $c_{1}, \ldots, c_{s}$ of $G$ will be called an irreducible covering of $G$ if the union of any $s-1$ cliques from $C$ is a proper subset of $V(G)$. This means that there exist $s$ vertices $x_{1}, \ldots, x_{s} \in V(G)$ that are uniquely covered by cliques of $C$, i.e., $x_{i} \notin \bigcup_{\substack{k=1 \\ k \neq i}}^{s} c_{k}$ for every $1 \leq i \leq s$.

If $G=K_{p, q}$, every clique of $G$ is an edge and an irreducible covering by edges of $K_{p, q}$ consists of a set of vertex-disjoint stars, some centered in the part with $p$ vertices and others in the part with $q$ vertices of $K_{p, q}$, which cover together all vertices of $K_{p, q}$. Some properties of the numbers $N(p, q)$ of all irreducible coverings by edges of $K_{p, q}$ were deduced in [8] and the exponential generating function of these numbers was given in [9]. Also, by denoting $I(n, n-k)$ the maximum number of irreducible coverings of the vertices of an $n$-vertex graph by $n-k$ cliques, in [8] it was shown that $\lim _{n \rightarrow \infty} I(n, n-k)^{1 / n}=\alpha(k)$, where $\alpha(k)$ is the greatest number of cliques a graph with $k$ vertices can have. The problem of determining $\alpha(k)$ was solved by Miller and Muller [2] and independently 
by Moon and Moser [3]. Furthermore, $I(n, n-2)=2^{n-2}-2$ and the extremal graph (unique up to isomorphism) coincides with $K_{2, n-2}$ for every $n \geq 4$. In [10] it was proved that for sufficiently large $n, I(n, n-3)=3^{n-3}-3 \cdot 2^{n-3}+3$, and the extremal graph is (up to isomorphism) $K_{3, n-3}$, the second extremal graph being $K_{3, n-3}-e$.

There is a class of algorithms which yield all irreducible coverings for the set-covering problem, an example of an algorithm in this class being Petrick's algorithm [5]. This algorithm was intensively used for obtaining the minimal disjunctive forms of a Boolean function using prime implicants of the function or for minimizing the number of states of an incompletely specified Mealy type automaton $A$ by finding a closed irreducible covering of the set of states of $A$ by "maximal compatible sets of states", which are cliques in the graph of compatible states of $A[4,7]$, since every minimum covering is an irreducible one. The chromatic number $\chi(G)$ of $G$ equals the minimum number of cliques from an irreducible covering by cliques of the complementary graph $\bar{G}$.

\section{Main result}

We will evaluate the clique number $\omega(G)$ when $G$ of order $n$ has an irreducible covering by $n-k$ cliques.

Theorem 2.1 Let $k \geq 2$. If the graph $G$ of order $n$ has an irreducible covering by $n-k$ cliques, then $\omega(G) \leq k+1$ if $k=2$ or 3 and $\omega(G) \leq\left(\begin{array}{c}k \\ \lfloor k / 2\rfloor\end{array}\right)$ if $k \geq 4$. Moreover, these bounds are sharp for every $n \geq k+1$ if $k=2$ or 3 and $n \geq k+\left(\begin{array}{c}k \\ \lfloor k / 2\rfloor\end{array}\right)$ if $k \geq 4$.

Proof: Let $C=\left\{c_{1}, \ldots, c_{n-k}\right\}$ be an irreducible covering by $n-k$ cliques of $G$. It follows that there are $n-k$ vertices $x_{1}, \ldots, x_{n-k} \in V(G)$ such that $x_{i} \in c_{i} \backslash \bigcup_{j \neq i} c_{j}$ for every $i=1, \ldots, n-k$. Denoting $X=\left\{x_{1}, \ldots, x_{n-k}\right\}$ and $Y=V(G) \backslash X$ one has $|Y|=k$. Each clique $c_{i}$ consists of $x_{i}$ and a subset of $Y$. For every subset $A \subseteq Y$ let $X_{A} \subseteq X$ be defined by

$$
X_{A}=\left\{x_{i} \in X: c_{i}=\left\{x_{i}\right\} \cup A\right\} .
$$

It is clear that if $x_{i}, x_{j} \in X_{A}$ then $x_{i} x_{j} \notin E(G)$ since otherwise $A \cup\left\{x_{i}, x_{j}\right\}$ induces a complete subgraph in $G$ whose vertex set contains strictly $c_{i}$ and $c_{j}$, which contradicts the definition of a clique. Similarly, if $x_{i} \in X_{A}, x_{j} \in X_{B}$ and $A \subset B$ it follows that $x_{i} x_{j} \notin$ $E(G)$ since otherwise $A \cup\left\{x_{i}, x_{j}\right\}$ induces a complete subgraph in $G$, thus contradicting the hypothesis that $c_{i}$ is a clique.

This implies that each clique $c$ in $G$ has the form $\left\{t_{1}, \ldots, t_{s}\right\} \cup \bigcap_{i=1}^{s} A_{i}$ for some $s \geq 2$, where $X_{A_{i}} \neq \emptyset, t_{i} \in X_{A_{i}} \subset X$ for every $1 \leq i \leq s$ and $\left\{A_{1}, \ldots, A_{s}\right\}$ is an antichain in the poset of subsets of $Y$, or $c$ induces a maximal complete subgraph with vertex set included in $Y \cup\left\{x_{i}\right\}$ for some $1 \leq i \leq n-k$.

We will show for the first case that

$$
\max _{s \geq 2} \max _{\left\{A_{1}, \ldots, A_{s}\right\}}\left(s+\left|\bigcap_{i=1}^{s} A_{i}\right|\right)=\left(\begin{array}{c}
k \\
\lfloor k / 2\rfloor
\end{array}\right),
$$


where the second maximum in the left-hand side of (1) is taken over all antichains of length $s \geq 2,\left\{A_{1}, \ldots, A_{s}\right\}$ in the poset of subsets of $Y(|Y|=k \geq 2)$, ordered by inclusion. The proof of (1) is by double inequality. If we choose $\left\{A_{1}, \ldots, A_{s}\right\}$ to be the family of all $\lfloor k / 2\rfloor$-subsets of $Y$ we have $s=\left(\begin{array}{c}k \\ \lfloor k / 2\rfloor\end{array}\right)$ and $\bigcap_{i=1}^{s} A_{i}=\emptyset$, whence

$$
\max _{s \geq 2} \max _{\left\{A_{1}, \ldots, A_{s}\right\}}\left(s+\left|\bigcap_{i=1}^{s} A_{i}\right|\right) \geq\left(\begin{array}{c}
k \\
\lfloor k / 2\rfloor
\end{array}\right) \text {. }
$$

On the other hand, let $B=\bigcap_{i=1}^{s} A_{i}$ and $r=|B|$. Since $s \geq 2$ and $\left\{A_{1}, \ldots, A_{s}\right\}$ is an antichain, it follows that $r \leq k-2$. By deleting elements of $B$ from $A_{1}, \ldots, A_{s}$ we get an antichain in the poset of subsets of $Y \backslash B(|Y \backslash B|=k-r)$, ordered by inclusion. By Sperner's theorem [6] it follows that

$$
\max _{\left\{A_{1}, \ldots, A_{s}\right\}}\left(s+\left|\bigcap_{i=1}^{s} A_{i}\right|\right) \leq\left(\begin{array}{c}
k-r \\
\lfloor(k-r) / 2\rfloor
\end{array}\right)+r
$$

and the last expression is less than or equal to $\left(\begin{array}{c}k \\ \lfloor k / 2\rfloor\end{array}\right)$ for every $k \geq 2$ and $0 \leq r \leq k-1$ and (1) is proved. Since any maximal complete subgraph in $Y \cup\left\{x_{i}\right\}$ can have at most $k+1$ vertices, it follows that

$$
\omega(G) \leq \max \left(k+1,\left(\begin{array}{c}
k \\
\lfloor k / 2\rfloor
\end{array}\right)\right)
$$

i.e., $\omega(G) \leq k+1$ if $k=2$ or 3 and $\omega(G) \leq\left(\begin{array}{c}k \\ \lfloor k / 2\rfloor\end{array}\right)$ if $k \geq 4$.

If $k=2$ or $k=3$ we can consider a graph $G$ consisting of $n-k$ cliques of size $k+1$ each having a $k$-clique in common; then $G$ has order $n$, an irreducible covering by $n-k$ cliques and $\omega(G)=k+1$.

If $k \geq 4$ we define a graph $G$ of order $n \geq k+\left(\begin{array}{c}k \\ \lfloor k / 2\rfloor\end{array}\right)$ possessing an irreducible covering by $n-k$ cliques and $\omega(G)=\left(\begin{array}{c}k \\ \lfloor k / 2\rfloor\end{array}\right)$ as follows: Take a complete graph $K_{k}$ and other $n-k$ vertices $x_{1}, \ldots, x_{n-k}$. Let $A_{1}, \ldots, A_{p}$ with $p=\left(\begin{array}{c}k \\ k k / 2\rfloor\end{array}\right)$ be all subsets of $V\left(K_{k}\right)$ of cardinality $\lfloor k / 2\rfloor$. Since $n-k \geq p$, there is a partition $X=X_{1} \cup \ldots \cup X_{p}$ into $p$ classes of $X=\left\{x_{1}, \ldots, x_{n-k}\right\}$. Now join by an edge each vertex $x \in X_{i}$ to each vertex $y \in A_{i}$ for every $1 \leq i \leq p$ and add edges between some pairs of vertices in $X$ such that $X$ induce a complete multipartite graph whose parts are $X_{1}, \ldots, X_{p}$. This graph has an irreducible covering by $n-k$ cliques, clique number $\omega(G)=p=\left(\begin{array}{c}k \\ \lfloor k / 2\rfloor\end{array}\right)$ and $\prod_{i=1}^{p}\left|X_{i}\right|$ cliques with $p$ vertices.

\section{References}

[1] B. Bollobás, Modern Graph Theory, Springer-Verlag, New York, 1998. 
[2] R. E. Miller, D. E. Muller, A problem of maximum consistent subsets, IBM Research Report RC-240, J. T. Watson Research Center, Yorktown Heights, New York, 1960.

[3] J. W. Moon, L. Moser, On cliques in graphs, Israel J. of Math., 3(1965), 23-28.

[4] M. C. Paull, S. H. Unger, Minimizing the number of states in incompletely specified sequential functions, IRE Trans. Electronic Computers, Vol. EC-8 (1959), 356-367.

[5] S. R. Petrick, A direct determination of the irredundant forms of a Boolean function from the set of prime implicants, AFCRC-TR-56-110, Air Force Cambridge Research Center, 1956.

[6] E. Sperner, Ein Satz über Untermengen einer endlichen Menge, Math. Zeitschrift, 27(1928), 544-548.

[7] I. Tomescu, Combinatorial methods in the theory of finite automata (in French), Logique, Automatique, Informatique, 269-423, Ed. Acad. R.S.R., Bucharest, 1971.

[8] I. Tomescu, Some properties of irreducible coverings by cliques of complete multipartite graphs, J. of Combinatorial Theory, Series B, 2, 28(1980), 127-141.

[9] I. Tomescu, On the number of irreducible coverings by edges of complete bipartite graphs, Discrete Mathematics, 150(1996), 453-456.

[10] I. Tomescu, On the maximum number of irreducible coverings of an $n$-vertex graph by $n-3$ cliques, Computing and Combinatorics, Proceedings, 8th Annual Int. Conf., COCOON 2002, Singapore, August 2002, O. Ibarra, L. Zhang (Eds.), LNCS 2387, Springer (2002), 544-553. 